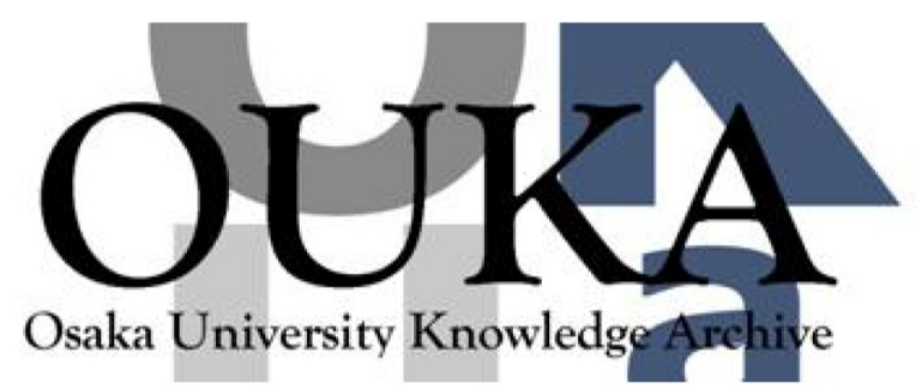

\begin{tabular}{|c|c|}
\hline Title & $\begin{array}{l}\text { High-resolution }\left({ }^{\wedge}\langle 3\rangle \mathrm{He}, \mathrm{t}\right) \text { reaction on the } \\
\text { double- } \beta \text { decaying nucleus }{ }^{\wedge}\langle 136\rangle \mathrm{Xe}\end{array}$ \\
\hline Author(s) & Puppe, P.; Frekers, D. ; Adachi, T. et al. \\
\hline Citation & $\begin{array}{l}\text { Physical Review C - Nuclear Physics. 84(5) } \\
\text { p. } 51305-\text { p. } 51305\end{array}$ \\
\hline Issue Date & $2011-11-15$ \\
\hline oaire:version & VoR \\
\hline URL & https://hdl. handle. net/11094/51205 \\
\hline rights & ๑2011 American Physical Society \\
\hline Note & \\
\hline
\end{tabular}

Osaka University Knowledge Archive : OUKA

https://ir. Library. osaka-u. ac. jp/

Osaka University 


\title{
High-resolution $\left({ }^{3} \mathrm{He}, t\right)$ reaction on the double- $\beta$ decaying nucleus ${ }^{136} \mathrm{Xe}$
}

\author{
P. Puppe, ${ }^{1}$ D. Frekers, ${ }^{1}$ T. Adachi, ${ }^{2}$ H. Akimune,${ }^{3}$ N. Aoi, ${ }^{4}$ B. Bilgier,${ }^{5}$ H. Ejiri, ${ }^{4}$ H. Fujita, ${ }^{4,6}$ Y. Fujita, ${ }^{6}$ M. Fujiwara, ${ }^{4}$ \\ E. Ganioğlu, ${ }^{5}$ M. N. Harakeh, ${ }^{7}$ K. Hatanaka, ${ }^{4}$ M. Holl, ${ }^{1}$ H. C. Kozer, ${ }^{5}$ J. Lee, ${ }^{8}$ A. Lennarz, ${ }^{1}$ H. Matsubara, ${ }^{9}$ \\ K. Miki, ${ }^{4}$ S. E. A. Orrigo, ${ }^{10}$ T. Suzuki, ${ }^{4}$ A. Tamii, ${ }^{4}$ and J. H. Thies ${ }^{1}$ \\ ${ }^{1}$ Institut für Kernphysik, Westfälische Wilhelms-Universität, D-48149 Münster, Germany \\ ${ }^{2}$ Research Center for Electron and Photon Science, Tohoku University, Sendai, Miyagi 982-0826, Japan \\ ${ }^{3}$ Department of Physics, Konan University, Kobe 658-8501, Japan \\ ${ }^{4}$ Research Center for Nuclear Physics, Osaka University, Ibaraki, Osaka 567-0047, Japan \\ ${ }^{5}$ Istanbul University, Science Faculty, Department of Physics, TR-34134 Vezneciler, Istanbul, Turkey \\ ${ }^{6}$ Department of Physics, Osaka University, Toyonaka, Osaka 560-0043, Japan \\ ${ }^{7}$ Kernfysisch Versneller Instituut, University of Groningen, NL-9747 AA Groningen, The Netherlands \\ ${ }^{8}$ RIKEN Nishina Center, Wako, Saitama 351-0198, Japan \\ ${ }^{9}$ Center for Nuclear Study, University of Tokyo, Hirosawa 2-1, Wako, Saitama 351-0198, Japan \\ ${ }^{10}$ Instituto de Física Corpuscular, CSIC-Universidad de Valencia, E-46071 Valencia, Spain
}

(Received 3 September 2011; published 15 November 2011)

\begin{abstract}
A $\left({ }^{3} \mathrm{He}, t\right)$ charge-exchange reaction experiment on the double-beta decaying nucleus ${ }^{136} \mathrm{Xe}$ has been performed at an incident energy of $420 \mathrm{MeV}$ with the objective to measure the Gamow-Teller (GT) strength distribution in ${ }^{136} \mathrm{Cs}$. The measurements have been carried out at the dispersion-matched WS beam line and the Grand Raiden spectrometer of the Research Center for Nuclear Physics in Osaka, where an energy resolution of $42 \mathrm{keV}$ was achieved. A new gas cell with thin windows made of polyethylene naphthalate has been employed as a target. The extracted GT strength distribution is confronted with the rather long $2 \nu \beta \beta$ decay half-life of ${ }^{136} \mathrm{Xe}$.
\end{abstract}

DOI: 10.1103/PhysRevC.84.051305

PACS number(s): $25.55 . \mathrm{Kr}, 23.40 . \mathrm{Hc}, 27.60 .+\mathrm{j}$

Introduction. In the context of the $\beta \beta$ decay, ${ }^{136} \mathrm{Xe}$ is a rather special system. From systematics, i.e., by looking at neighboring $\beta \beta$ decaying nuclei, its $2 v$ half-life would not be expected to be significantly longer than $T_{1 / 2}^{2 v} \approx 10^{20} \mathrm{yr}$. This is a value which is mostly supported by theory as well [1-6], although in Ref. [7] some arguments for a longer half-life relating this to the $N=82$ neutron shell closure have been provided. The relatively high $\beta \beta$ decay $Q$ value $(Q=2.467 \mathrm{MeV})$ and the high $Z$ value $(Z=54)$ are advantageous and ought to have a significant bearing on the value of the decay time. Yet, for a long time, the $2 v \beta \beta$ decay has evaded detection [8-12], and only recently the EXO-200 collaboration successfully measured the halflife to $T_{1 / 2}^{2 v}=[2.11 \pm 0.04$ (stat.) \pm 0.21 (sys.) $] \times 10^{21} \mathrm{yr}(90 \%$ C.L.) [13]. Accordingly, the value for the nuclear matrix element was quoted as $M_{\text {DGT }}^{2 v}=0.019 \mathrm{MeV}^{-1}$, which is the smallest one ever measured. This means that the various matrix elements, which connect the intermediate states in the ${ }^{136} \mathrm{Cs}$ intermediate nucleus must also either be small or there is some fortuitous phase cancellation among the various components at work, which suppresses the decay rather effectively. Experimental charge-exchange data on ${ }^{136} \mathrm{Xe}$, which could shed light on the size of these matrix elements do not exist, and even more astounding, not even a single excited state in the intermediate nucleus ${ }^{136} \mathrm{Cs}$ is known [14]. Nonetheless, counting experiments on ${ }^{136} \mathrm{Xe}$ searching for the neutrinoless $(0 \nu \beta \beta)$ decay are planned as well, as, e.g., by the EXO collaboration, or more recently by KamLAND-ZeN [15-19]. Assuming that the $2 \nu \beta \beta$ decays and the $0 \nu \beta \beta$ decays are not strongly correlated, a low $2 v \beta \beta$ decay rate is then rather advantageous for these experiments, as it would constitute a much reduced background in the vicinity of the endpoint energy [20].

The present Rapid Communication aims at providing a much warranted experimental input into the structure of the $A=136$ system by using the $\left({ }^{3} \mathrm{He}, t\right)$ charge-exchange reaction at intermediate energies. Charge-exchange reactions into the $\beta^{-}$direction [as, e.g., $\left({ }^{3} \mathrm{He}, t\right)$ or $\left.(p, n)\right]$ or $\beta^{+}$ direction [as, e.g., $\left(t,{ }^{3} \mathrm{He}\right),\left(d,{ }^{2} \mathrm{He}\right)$ or $\left.(n, p)\right]$ are an established tool for extracting Gamow-Teller (GT) transition strengths and are therefore rather effective to provide information about the $\mathrm{GT}^{-}$and $\mathrm{GT}^{+}$parts of the nuclear matrix elements of the $2 v \beta \beta$ decay [21-33]. The selectivity is a result of the dominance of the $\vec{\sigma} \overrightarrow{\boldsymbol{\tau}}$ effective nucleon-nucleon interaction at intermediate energies and low momentum transfers [34-37].

It may be instructive to recall the connection between the $2 v \beta \beta$ decay nuclear matrix element and the $2 v \beta \beta$ decay halflife:

$$
\left(T_{1 / 2}^{2 v}\right)^{-1}=G^{2 v}(Q, Z)\left|M_{\mathrm{DGT}}^{2 v}\right|^{2}
$$

and

$$
M_{\mathrm{DGT}}^{2 v}=\sum_{m} \frac{M_{m}\left(\mathrm{GT}^{+}\right) \cdot M_{m}\left(\mathrm{GT}^{-}\right)}{1 / 2 Q_{\beta \beta}\left(0_{\text {g.s. }}^{(f)}\right)+E_{x}\left(1_{m}^{+}\right)-E_{0}} .
$$

Here, $E_{x}\left(1_{m}^{+}\right)-E_{0}$ is the energy difference between the $m$ th intermediate $1^{+}$state and the initial ground state, and $M_{m}\left(\mathrm{GT}^{ \pm}\right)$are the single $\beta$-decay matrix elements in the $\beta^{+}$ and $\beta^{-}$directions, respectively. [Note that the insertion of $M_{m}\left(\mathrm{GT}^{+}\right)$is the result of time invariance; also note that the energy denominator is often expressed in units of the electron 
rest mass $m_{e}$.] The quantity $G^{2 v}(Q, Z)$ is the phase-space factor, and values for different nuclei are summarized in Ref. [38].

Because the relation between the GT transition strength and the single $\beta$-decay matrix element for a $0^{+} \rightarrow 1^{+}$transition is

$$
B_{m}\left(\mathrm{GT}^{ \pm}\right)=\left|M_{m}\left(\mathrm{GT}^{ \pm}\right)\right|^{2},
$$

sign properties, which are important for the summation in Eq. (2), get lost when deriving the latter from the former.

Experiment. The experiment was performed at the Research Center for Nuclear Physics (RCNP) in Osaka. A $420-\mathrm{MeV}^{3} \mathrm{He}$ beam was accelerated and focused onto a gas target located in the scattering chamber at the entrance of the Grand Raiden spectrometer. The WS beam line [39] provided the necessary dispersion of the beam for achieving the highest resolution [40]. Several tuning techniques for the dispersion matching between beam line and the spectrometer were employed to optimize energy and angular resolution [41-43].

A specially designed gas-target system [44] was employed to provide a target cell with isotopically enriched ${ }^{136} \mathrm{Xe}$ $(99.92 \%)$ gas at the desired areal density. The gas cell was made of a $6 \mathrm{~mm}$ thick copper piece with an inner 2.8-mm-diam gas support line and an open area sealed by $6-\mu \mathrm{m}$-thin polyethylene naphthalate (PEN) (trademark Teonex ${ }^{\circledR}$ Q51) entrance and exit window foils. The windows were $\sim 30 \mathrm{~mm}$ wide and $7 \mathrm{~mm}$ high. The physical thickness of the target was $6 \mathrm{~mm}$. Since the vertical extent of the dispersed beam was significantly less than $1 \mathrm{~mm}$, background due to slit scattering did not occur during the experiment. The foils were produced by DuPont Teijin Films and only contain carbon, hydrogen, and oxygen components. Whereas hydrogen never presents a serious background, the natural ${ }^{13} \mathrm{C}$ and ${ }^{18} \mathrm{O}$ components, though of low natural abundance, have a low enough reaction $Q$ value $\left(Q_{R}=2.239\right.$ and $\left.1.674 \mathrm{MeV}\right)$ to cause a sizable background in the region of low excitations of the ${ }^{136} \mathrm{Xe}$ reaction $\left(Q_{R}=0.108 \mathrm{MeV}[45]\right)$. The ${ }^{12} \mathrm{C}$ and ${ }^{16} \mathrm{O}$ ground-state transitions, on the other hand, appear at 17.248 and 15.327 MeV in the excitation-energy frame of ${ }^{136} \mathrm{Xe}$ and do therefore not contaminate the low-energy region. Empty target cell measurements with high statistics allowed an off-line subtraction of the contributions from the window foils.

The gas cell was connected to a computer-controlled gas supply system that provided the ${ }^{136} \mathrm{Xe}$ gas at the desired pressure of $0.5 \mathrm{~atm}$ at room temperature. This pressure constitutes an areal density of the target of $\sim 2.2 \mathrm{mg} / \mathrm{cm}^{2}$. Throughout the experiment the pressure stability and the temperature were monitored by a precision pressure gauge and a temperature sensor (PT100) installed inside the copper frame of the cell. The gas pressure caused a slight bulging of the foils, thereby increasing the areal density of the gas target. The effect was measured using the $\left({ }^{3} \mathrm{He}, t\right)$ reaction on ${ }^{12} \mathrm{C}$ by filling the cell with $\mathrm{CO}_{2}$ to equal pressure and temperature and normalizing the yield to the known cross section of the transition to the ${ }^{12} \mathrm{~N}$ ground state.

The PEN windows were found to safely withstand a $4 \mathrm{nA}$ electrical current at $420 \mathrm{MeV}$ for more than $30 \mathrm{~h}$. Several cells were used during the course of the experiment.

A precise energy calibration at the level of $\pm 5 \mathrm{keV}$ accuracy was performed using a solid natural zirconium target containing some minor contamination of natural silicon and carbon. The various known $\mathrm{Zr}, \mathrm{Si}$, and $\mathrm{C}$ lines in the spectrum span an excitation-energy region from 0 to $\sim 17 \mathrm{MeV}$ in the excitation-energy frame of the ${ }^{136} \mathrm{Cs}$ final nucleus.

The experiment was performed at two spectrometer-angle settings, i.e., $0^{\circ}$ and $2.5^{\circ}$. Appropriate solid angle cuts allowed generating angular distributions ranging from $\sim 0^{\circ}$ to $4.0^{\circ}$.

After applying various off-line spectrometer aberration corrections (up to tenth order) a final-state energy resolution

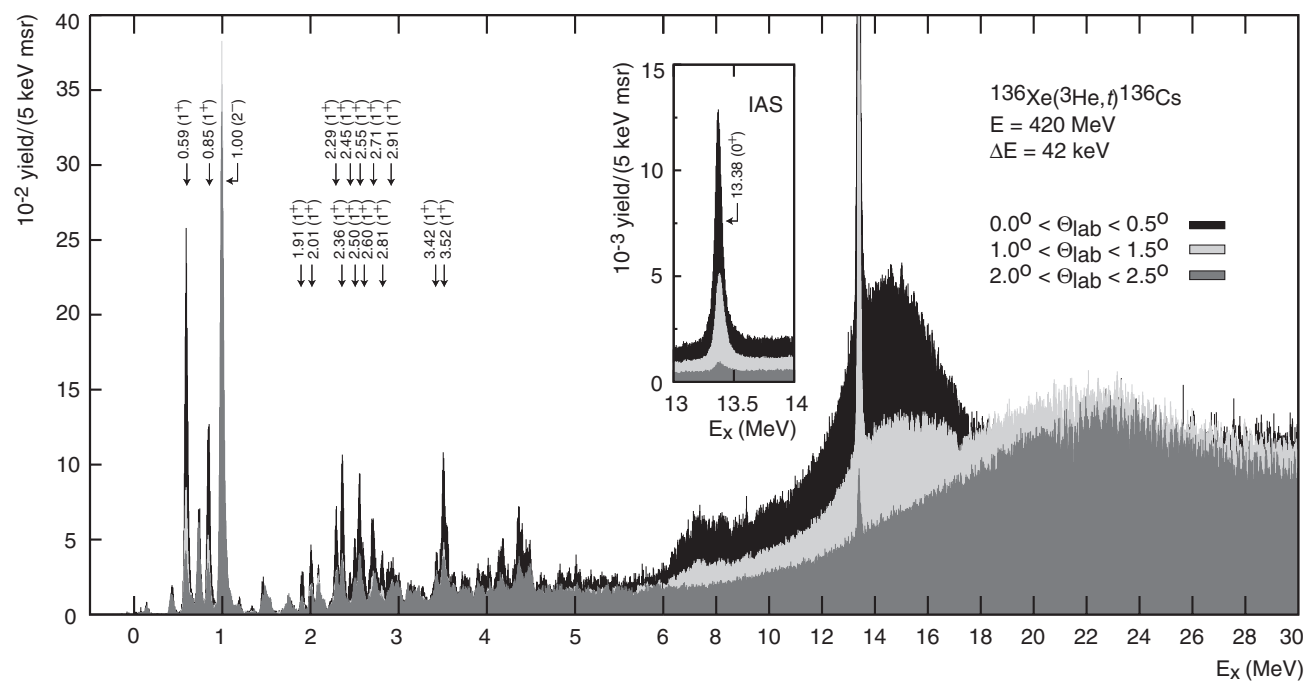

FIG. 1. Excitation-energy spectra of the ${ }^{136} \mathrm{Xe}\left({ }^{3} \mathrm{He}, t\right){ }^{136} \mathrm{Cs}$ reaction. Backgrounds from the window foils of the gas cell are subtracted. The spectra were generated for different angle cuts (as indicated by the color/shade) and stacked on top of each other to indicate the effect of the angular dependence. GT transitions are forward peaked and appear in black color at the most forward angle. Note that the energy scale is compressed above $6 \mathrm{MeV}$. Whereas the $J^{\pi}$ assignments for the states indicated as $1^{+}$and the one for the IAS are considered unique by the shape of the angular distribution, the one indicated as $2^{-}$may still be considered tentative. 

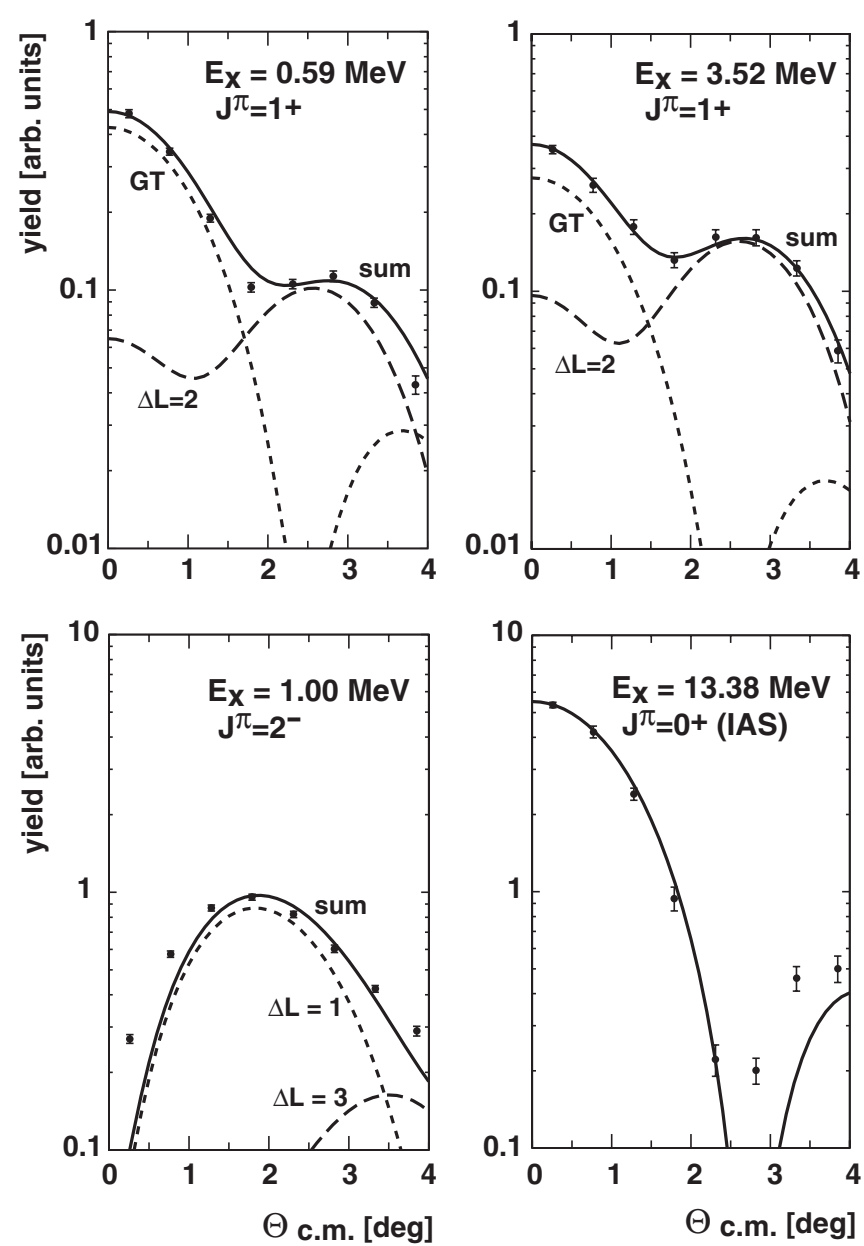

FIG. 2. Angular distributions of typical $1^{+}, 2^{-}$, and $0^{+}$(IAS) transitions. The error bars denote the combined statistical and systematic uncertainties. The lines illustrate DWBA calculations for the various components.

of $\sim 42 \mathrm{keV}$ was obtained for the ${ }^{136} \mathrm{Xe}$ target. This value is largely determined by the target areal thickness and its physical extent in the direction of the beam.

Analysis. Figure 1 shows three spectra of the ${ }^{136} \mathrm{Xe}\left({ }^{3} \mathrm{He}, t\right)$ ${ }^{136} \mathrm{Cs}$ reaction at three different forward scattering angles. The spectra have been arranged in such a way that the most forwardangle spectrum appears in the back and the others are stacked on top of each other using different color codings (black, white, and gray). This way the angular dependence of the various components can be quickly identified; e.g., forward-peaked cross sections are likely GT transitions (colored black) with angular momentum transfer $\Delta L=0$ and more backward peaked ones are likely $\Delta L=1$ spin-dipole transitions (colored gray). The spectra show a moderate number of isolated states up to $\sim 4.5 \mathrm{MeV}$ excitation energy, followed by the strongly excited isobaric analog state (IAS) at $13.38 \mathrm{MeV}$. At $\sim 15 \mathrm{MeV}$ one observes the peak of the giant GT resonance and at $\sim 23 \mathrm{MeV}$ the rather broad $(\Gamma \simeq 10 \mathrm{MeV})$ spin-dipole resonance.

Angular distributions were extracted for the transitions indicated in Fig. 1 and compared with distorted-wave Born approximation (DWBA) calculations performed with the codes NORMOD [46] and FOLD [47]. Appropriate optical model parameters were adapted from those of ${ }^{90} \mathrm{Zr}$ as quoted in Ref. [48]. Angular distributions for the two strongest $1^{+}$states at 0.59 and $3.52 \mathrm{MeV}$, the strong state at $1.00 \mathrm{MeV}$ (assumed to have $J^{\pi}=2^{-}$), and the IAS at $13.38 \mathrm{MeV}$ are shown in Fig. 2 and compared with DWBA calculations. In order to arrive at a good description of the experimental angular distributions, we find that for all $1^{+}$states there is a need to add a significant $\Delta L=2$ angular momentum transfer component to the cross section, which is something that may be related to the high density of unoccupied shells near the Fermi surface. This component should add coherently to the cross section, however, due to the lack of a realistic ${ }^{136} \mathrm{Xe}$ wave function, one is simply left to add it in an incoherent way. The procedure may receive some justification by the good description one is able to achieve.

There are numerous ways to extract the GT strength from the angular distribution. All of them relate the GT part of the cross section to the weak decay strength by extrapolating the angular distribution to zero momentum transfer $(q=0)$. The functional form of this extrapolation is given by the zeroth-order Bessel function $\left|j_{0}\left(q R_{0}\right)\right|^{2}$, with $R_{0}$ being the interaction radius (see, e.g., Ref. [37]). This extrapolation is in accord with the standard plane-wave approach and may not be an appropriate description for the hadronic reaction, as this requires incoming distorted Coulomb waves.

In the present Rapid Communication we have used a method whereby we combine the Gamow-Teller and Fermi unit cross sections at $q=0$ to extract the GT strength:

$$
R^{2}(E, A)=\frac{\hat{\sigma}_{\mathrm{GT}}}{\hat{\sigma}_{F}}=\frac{\sigma_{\mathrm{GT}}(q \rightarrow 0)}{B\left(\mathrm{GT}^{-}\right)} / \frac{\sigma_{F}(q \rightarrow 0)}{B(\mathrm{~F})} .
$$

Following Refs. [49-51], $R^{2}$ takes a value of $11.0 \pm 1.0$ in this mass range and $B(\mathrm{~F})=28$ for ${ }^{136} \mathrm{Xe}$. The $B\left(\mathrm{GT}^{-}\right)$values extracted according to this recipe are listed in Table I. Because of the uncertainty with which the extra non-GT contribution can be evaluated at $q=0$, we suppose that $50 \%$ of the non-GT part to the cross section at $q=0$ (see, e.g., the second columns in Table I) should enter into the error calculation for the strength values. Such a conservative error margin would then also include possible non-GT tensor contributions to the cross

TABLE I. Excitation energies of $1^{+}$states populated in the ${ }^{136} \mathrm{Xe}\left({ }^{3} \mathrm{He}, t\right){ }^{136} \mathrm{Cs}$ reaction. The second columns indicate what percentage of the cross section at $q=0$ can be attributed to a GT transition using the extraction procedure as described in the text. The extracted $B(\mathrm{GT})$ values are given in columns 3 .

\begin{tabular}{lclccl}
\hline \hline $\begin{array}{l}E_{x} \\
(\mathrm{MeV})\end{array}$ & $\begin{array}{c}\mathrm{GT} \\
\text { fraction }\end{array}$ & $B\left(\mathrm{GT}^{-}\right)$ & $\begin{array}{c}E_{x} \\
(\mathrm{MeV})\end{array}$ & $\begin{array}{c}\mathrm{GT} \\
\text { fraction }\end{array}$ & $B\left(\mathrm{GT}^{-}\right)$ \\
\hline 0.59 & $87 \%$ & $0.149(21)$ & 2.55 & $66 \%$ & $0.033(11)$ \\
0.85 & $81 \%$ & $0.082(15)$ & 2.60 & $78 \%$ & $0.033(7)$ \\
1.91 & $72 \%$ & $0.017(5)$ & 2.71 & $66 \%$ & $0.055(18)$ \\
2.01 & $66 \%$ & $0.024(8)$ & 2.81 & $71 \%$ & $0.026(7)$ \\
2.29 & $73 \%$ & $0.042(11)$ & 2.91 & $58 \%$ & $0.027(12)$ \\
2.36 & $72 \%$ & $0.059(15)$ & 3.42 & $59 \%$ & $0.024(10)$ \\
2.45 & $74 \%$ & $0.015(4)$ & 3.52 & $74 \%$ & $0.097(23)$ \\
2.50 & $66 \%$ & $0.024(8)$ & & & $\sum=0.71(1)$ \\
\hline \hline
\end{tabular}



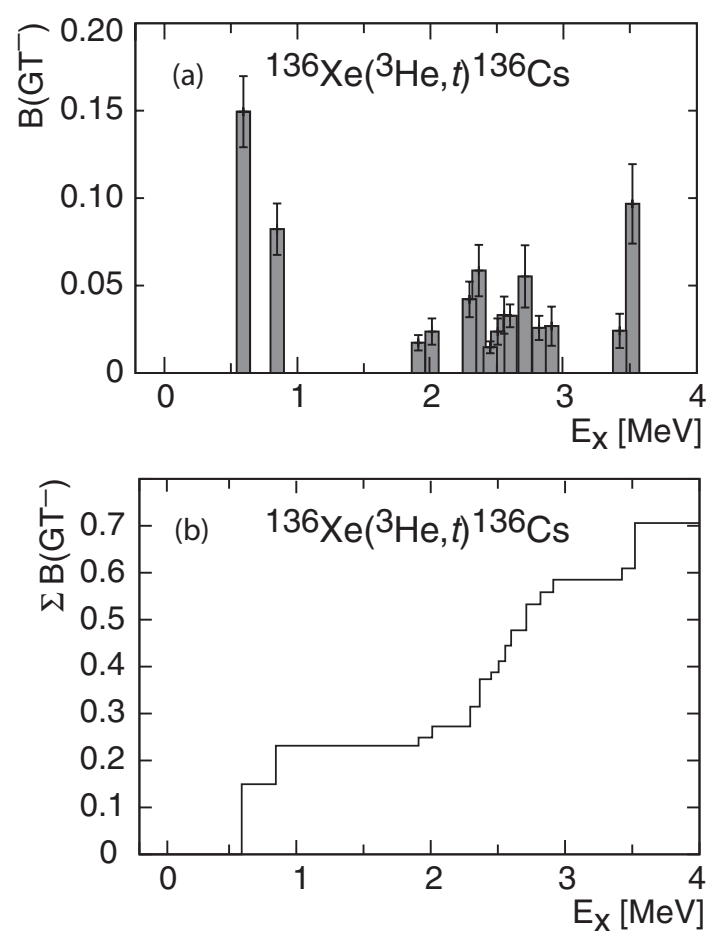

FIG. 3. (a) Distribution of $B\left(\mathrm{GT}^{-}\right)$strength in the low excitationenergy region of the ${ }^{136} \mathrm{Xe}\left({ }^{3} \mathrm{He}, t\right){ }^{136} \mathrm{Cs}$ reaction; (b) running sum of the $B\left(\mathrm{GT}^{-}\right)$strength.

section. The $B(\mathrm{GT})$ strength values up to $\sim 4 \mathrm{MeV}$ extracted in this way are displayed in Fig. 3 together with the running sum, which yields a value of $\sum_{0-3.5 \mathrm{MeV}} B\left(\mathrm{GT}^{-}\right)=0.71$.

Connection to $\beta \beta$ decay. In Refs. [23,24,29-32] it was reported that the low-lying $\mathrm{GT}^{-}$and $\mathrm{GT}^{+}$parts of the GT strength (up to $\sim 5 \mathrm{MeV}$ ) contribute a substantial fraction to the $\beta \beta$ nuclear matrix elements of Eq. (2). Although the higherlying giant $\mathrm{GT}^{-}$resonance is the most prominent component in typical $(p, n)$-type spectra [as is also nicely exemplified in Fig. 1 for the present $\left({ }^{3} \mathrm{He}, t\right)$ reaction], the resonance is conspicuously absent for a charge-exchange reaction in the $\mathrm{GT}^{+}$direction [29-32], thereby contributing comparatively little to the nuclear matrix elements. This absence is, of course, a rather trivial effect of Pauli blocking, since in a nucleus with a large neutron excess the neutron shells in the vicinity of the Fermi surface always find a largely unoccupied proton shell, whereas this is not the case for protons.

Therefore, the moderately strong low-lying $\mathrm{GT}^{-}$transitions appearing in the present ${ }^{136} \mathrm{Xe}$ charge-exchange spectra do not point to any special properties of ${ }^{136} \mathrm{Xe}$ in terms of an unusually strong suppression of GT strength. Several other possible scenarios may therefore be brought to bear for an explanation of the rather long half-life. Since the present $\left({ }^{3} \mathrm{He}, t\right)$ reaction only defines the $\mathrm{GT}^{-}$part, one could speculate about an exceptionally weak $\mathrm{GT}^{+}$part, which certainly would be something one could extract from a similar charge-exchange reaction [here $(n, p)$ type] on the $\beta \beta$-decay daughter ${ }^{136} \mathrm{Ba}$. Given the quoted number for the ${ }^{136} \mathrm{Xe}$ nuclear matrix element $M_{\text {DGT }}^{2 v}=0.019 \mathrm{MeV}^{-1}[13]$, the $B\left(\mathrm{GT}^{+}\right)$values for the states listed in Table I should then be on average in the range of $10^{-3}-10^{-2}$ times the $B\left(\mathrm{GT}^{-}\right)$values. Such a situation would indeed be unexpected and special.

Alternatively, there is the possibility of a rather effective phase cancellation in Eq. (2), which is something already conjectured in Ref. [29] for the ${ }^{48} \mathrm{Ca}$ case. In Fig. 3, we note a clustering of GT transitions between 0 and $1 \mathrm{MeV}$ and between 2 and $3.5 \mathrm{MeV}$. If the nuclear matrix elements have different signs for these two clusters, the elements under the sum in Eq. (2) could effectively cancel, especially if the $B(\mathrm{GT})$ values for the $\mathrm{GT}^{+}$and the $\mathrm{GT}^{-}$directions were moderately correlated. This can also easily be read off from the running sum in the lower part of Fig. 3.

Whereas the first scenario could be readily tested experimentally, the second one requires a detailed theoretical understanding of what the underlying physics is, which would cause certain matrix elements to have different signs. This may be an important aspect, since theoretical quasiparticle random phase approximation (QRPA)-based calculations [1-3,52-57] often use the $2 \nu \beta \beta$ decay half-life to adjust the particle-particle interaction parameter $g_{\mathrm{pp}}$ for predicting the $0 \nu \beta \beta$ decay nuclear matrix element, irrespective of possible cancellation effects.

Conclusion. We have used a high-resolution $\left({ }^{3} \mathrm{He}, t\right)$ chargeexchange reaction at $420 \mathrm{MeV}$ on the $\beta \beta$ decaying nucleus ${ }^{136} \mathrm{Xe}$ to identify the $\mathrm{GT}^{-}$strength distribution in the intermediate nucleus ${ }^{136} \mathrm{Cs}$ in an attempt to understand the long $2 \nu \beta \beta$ decay half-life of ${ }^{136} \mathrm{Xe}$. We find that the $\mathrm{GT}^{-}$ strength distribution even at low excitation energies exhibits a rather normal behavior. A number of well-isolated states up to $\sim 4 \mathrm{MeV}$, which are concentrated in two separate clusters, have been resolved carrying a total $B\left(\mathrm{GT}^{-}\right)$strength of 0.71 . We have argued that exceptionally weak transitions from the $\mathrm{GT}^{+}$ side would be a rather improbable cause for the long half-life, whereas phase-cancellation effects for the $\beta \beta$-decay nuclear matrix elements seem to be a more natural and likely scenario.

Acknowledgments. We thank the RCNP accelerator staff for their fine technical support during the course of the experiment. The generous financial support from the Directorate of RCNP is gratefully acknowledged. Y.F. and A.T. were partly supported by MEXT, Japan under Grant No. 22540310. S.E.A.O. was supported under the Spanish MICINN Grant No. FPA200806419-C02-01. The work was partly financed by the Deutsche Forschungsgemeinschaft (DFG) under Grant No. FR 601/3-1.
[1] P. Vogel and M. R. Zirnbauer, Phys. Rev. Lett. 57, 3148 (1986).
[2] A. Staudt, K. Muto, and H. V. Klapdor-Kleingrothaus, Europhys. Lett. 13, 31 (1990). 
[3] F. Šimkovic, A. Faessler, H. Müther, V. Rodin, and M. Stauf, Phys. Rev. C 79, 055501 (2009).

[4] E. Caurier, F. Nowacki, A. Poves, and J. Retamosa, Nucl. Phys. A 654, 973c (1999).

[5] E. Caurier, F. Nowacki, and A. Poves, Int. J. Mod. Phys. E 16, 552 (2007).

[6] E. Caurier, J. Menéndez, F. Nowacki, and A. Poves, Phys. Rev. Lett. 100, 052503 (2008).

[7] H. Ejiri, J. Phys. Soc. Jpn. 78, 074201 (2009).

[8] E. Bellotti, O. Cremonesi, E. Fiorini, G. Gervasio, S. Ragazzi, and L. Rossi, Phys. Lett. B 266, 193 (1991).

[9] R. Luescher, J. Farine, F. Boehm, J. Busto, K. Gabathuler, and G. Gervasio, Phys. Lett. B 434, 407 (1998).

[10] R. Bernabei, P. Belli, F. Cappella, R. Cerulli, F. Montecchia, and A. Incicchitti, Phys. Lett. B 546, 23 (2002).

[11] J. M. Gavriljuk, V. V. Kuzminov, N. Ya. Osetrova, and S. S. Ratkevich, Phys. Rev. C 61, 035501 (2000).

[12] J. M. Gavriljuk, A. M. Gangapshev, V. V. Kuzminov, S. I. Panasenko, and S. S. Ratkevich, Phys. At. Nucl. 69, 2129 (2006).

[13] N. Ackerman et al., arXiv:1108.4193v1.

[14] National Nuclear Data Center, Brookhaven National Laboratory (2011) [http://www.nndc.bnl.gov].

[15] M. Danilov et al., Phys. Lett. B 480, 12 (2000).

[16] D. Akimov, G. Bower, M. Breidenbach, R. Conley, and E. Conti, Nucl. Phys. Proc. Suppl. B 138, 224 (2005).

[17] R. Gornea, J. Phys. Conf. Ser. 259, 012039 (2010).

[18] EXO collaboration (2010) [http://www-project.slac.stanford. edu/exo/].

[19] Y. Efremenko, talk given at MEDEX'11-Conference on Matrix Elements for the Double-beta decay Experiments, Prague, June 13-16, 2011 (unpublished).

[20] H. Ejiri, Prog. Part. Nucl. Phys. 40, 307 (1998).

[21] W. P. Alford et al., Nucl. Phys. A 514, 49 (1990).

[22] H. Akimune et al., Phys. Lett. B 394, 23 (1997).

[23] H. Ejiri, Phys. Rep. 338, 265 (2000).

[24] H. Ejiri, J. Phys. Soc. Jpn. 74, 2101 (2005).

[25] G. W. Hitt et al., Nucl. Instrum. Methods Phys. Res. A 566, 264 (2006).

[26] S. Rakers et al., Nucl. Instrum. Methods Phys. Res. A 481, 253 (2002).

[27] S. Rakers et al., Phys. Rev. C 70, 054302 (2004).
[28] S. Rakers et al., Phys. Rev. C 71, 054313 (2005).

[29] E.-W. Grewe et al., Phys. Rev. C 76, 054307 (2007).

[30] E.-W. Grewe et al., Phys. Rev. C 77, 064303 (2008).

[31] E.-W. Grewe et al., Phys. Rev. C 78, 044301 (2008).

[32] H. Dohmann et al., Phys. Rev. C 78, 041602 (2008).

[33] C. J. Guess et al., Phys. Rev. C 83, 064318 (2011).

[34] W. G. Love and M. A. Franey, Phys. Rev. C 24, 1073 (1981).

[35] M. A. Franey and W. G. Love, Phys. Rev. C 31, 488 (1985).

[36] C. D. Goodman et al., Phys. Rev. Lett. 44, 1755 (1980).

[37] T. N. Taddeucci et al., Nucl. Phys. A 469, 125 (1987).

[38] J. Suhonen and O. Civitarese, Phys. Rep. 300, 123 (1998).

[39] T. Wasaka et al., Nucl. Instrum. Methods Phys. Res. A 482, 79 (2002).

[40] M. Fujiwaraet al., Nucl. Instrum. Methods Phys. Res. A 422, 484 (1999).

[41] Y. Fujita et al., Nucl. Instrum. Methods Phys. Res. B 126, 274 (1997).

[42] H. Fujita et al., Nucl. Instrum. Methods Phys. Res. A 469, 55 (2001).

[43] H. Fujita et al., Nucl. Instrum. Methods Phys. Res. A 484, 17 (2002).

[44] H. Matsubara, A. Tamii, T. Adachi, M. Dozono, H. Fujita, and K. Fujita, Nucl. Instrum. Methods (to be submitted, 2011).

[45] G. Audi and M. Wang (private communication).

[46] M. A. Hofstee et al., Nucl. Phys. A 588, 729 (1995).

[47] J. Cook and J. A. Carr, computer code FOLD, Florida State University, 1982 (unpublished).

[48] J. Kamiya et al., Phys. Rev. C 67, 064612 (2003).

[49] T. Adachi, Ph.D. thesis, Department of Physics, Osaka University, 2007.

[50] T. Adachi et al., Nucl. Phys. A 788, 70c (2007).

[51] Y. Fujita et al., in RCNP, Annual Report 2010.

[52] V. A. Rodin, A. Faessler, F. Šimkovic, and P. Vogel, Phys. Rev. C 68, 044302 (2003).

[53] V. A. Rodin, A. Faessler, F. Šimkovic, and P. Vogel, Nucl. Phys. A 766, 107 (2006); 793, 213(E) (2007).

[54] F. Šimkovic, A. Faessler, V. A. Rodin, P. Vogel, and J. Engel, Phys. Rev. C 77, 045503 (2008).

[55] J. Suhonen, Phys. Lett. B 607, 87 (2005).

[56] J. Suhonen and O. Civitarese, Phys. Lett. B 626, 80 (2005).

[57] J. Suhonen and O. Civitarese, Nucl. Phys. A 761, 313 (2005). 